\title{
Neuropharmacology for a new era
}

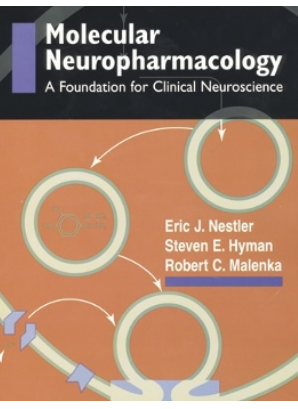

\section{Molecular Neuropharmacology: A Foundation for Clinical Neuroscience}

by Eric J. Nestler, Steven E. Hyman and

Robert C. Malenka

McGraw-Hill, New York, 2001. \$49.95

paperback, pp 539

ISBN 0-83-856379-1

Reviewed by Zach W. Hall
One of the hopes for future brain research is that we can translate our remarkable progress in fundamental neuroscience into a biological understanding of human behavior and its disorders. Molecular Neuropharmacology: A Foundation for Clinical Neuroscience admirably sets the stage for this challenge. It does so by focusing on drugs, which historically have been powerful investigative tools in brain research, both as precise reagents for unraveling cellular mechanisms in the laboratory and as active clinical agents whose effects give insight into the biological basis of complex behaviors and their disorders. Over the last few decades, neuropharmacology has largely focused on identifying the specific proteins that act as drug targets. This book not only reflects the success of these efforts, but also the perspective of a new era, emphasizing the molecular and cellular circuits that mediate the effects of drugs on behavior, as well as the ability of drugs to exert their effects by changing these circuits.

As clinically trained psychiatrists who are also leaders in fundamental brain research, the three authors, Eric Nestler, Steve Hyman and Robert Malenka, bring impressive credentials to the task. The result is a remarkable book-comprehensive, detailed and authoritative - that makes a welcome addition to the top rank of neuroscience texts.

With its twin focus on drugs and disease, the book is aimed at two audiences: non-medical research students who need to learn about disease, and clinical

Zach Hall is at the University of California San Francisco, 513 Parnassus Avenue, S-101, Office of Research, San Francisco, California,

94143-0407, USA.

e-mail:zhall@research.ucsf.edu researchers and practitioners, who need to learn about the drugs that they use. The names of drugs and diseases are introduced wherever relevant, and are highlighted in boldface throughout. Boxes are used to elaborate specific points. Several boxes are superb: a succinct description of neurofibromatosis, a discussion of the limitations of animal models of depression, and a summary of small $G$ proteins, among others. The text is additionally enlivened by first-person descriptions of ADHD, Alzheimer's disease and Sol Snyder's first LSD trip.

The book is divided into three sections. The first, Fundamentals of Neuroscience, covers basic neuropharmacology, the cell biology of neurons and glia, electrical excitability of neurons and synaptic transmission. These are standard textbook chapters. Some are perfunctory and, as teaching tools, unremarkable, but all are notable for their accuracy and currency. Discussions include the latest interpretations and discoveries (even the most recent estimate of the number of genes in the human genome) smoothly integrated into the text. Two of the chapters, Signal Transduction Pathways in the Brain and Signaling to the Nucleus, are gems. They are clear, authoritative and well organized.

The second section, Neural Substrates of Drug Action, covers each of the neurotransmitter systems, as well as neurotrophic factors. In contrast to traditional texts that emphasize simpler, peripheral systems, the emphasis here is on the CNS.

The most valuable section of the book is the third, Neuropharmacology of Specific Neural Functions and Related Disorders; here, the special talents of the authors shine. This section, which occupies almost half the book, contains chapters on the autonomic nervous system and the neuroendocrine system, as well as chapters on disorders of movement, mood, addiction, cognition, sleep, attention, pain, memory, seizures and stroke. Not surprisingly, the chapters on addiction and memory, which are the authors' specialties, are superb, as are those on mood and emotion and on cognitive function and psychosis. The treatment of movement disorders is less satisfactory, as it treats extensively the neural sub-circuits involved, without giving a strong sense of the mechanisms for overall control of movement. Also, by treating the movement disorders of Parkinson's, amyotrophic lateral sclerosis (ALS) and Huntington's disease (surprisingly, omitting the spino-cerebellar ataxias) separately from Alzheimer's, the authors miss an opportunity to highlight current ideas on protein aggregation as a unifying feature of the neurodegenerative diseases. In this context, a more detailed account of mechanisms of proteolysis, specifically with respect to misfolded proteins might have usefully received more attention in the chapter describing mechanisms of protein synthesis.

I have two general suggestions for the next edition. First, an early chapter on the influence of structural biology and the genomic revolution on our thinking about drugs could provide valuable guidance for the future. These topics are mentioned only in passing, and, remarkably in a book devoted to drugs, I found only a single example of the three-dimensional structure of a protein. Second, I hope the undoubted success of the first edition will encourage the authors and the editors to invest in the second edition by improving the quality of the text and figures. Better editing, better choice of tables (do we really need to know the molecular mass of ionotropic receptor subunits?), and more and better figures would all improve the book. A more careful job of copy-editing could invigorate the sometimes flabby prose ("It was determined that...") and reduce the frequency of small errors (two in the first four sentences of Chapter 1).

But it seems mean-spirited to labor over minor defects in a book that is so generous with its gifts. The overall intelligence of the book, its complete command of contemporary neuroscience and its ability to link this knowledge with the clinical action of drugs make it required reading for anyone who wants to understand neuropharmacology and the neuroscience of the normal and diseased brain. The book will not only inform students, but will encourage the union of clinical understanding and basic biology, which its distinguished authors so ably represent. 